\title{
An Agent Based Voting System for E-Learning Course Selection Involving Complex Preferences
}

\author{
Ali M. Aseere, David E. Millard, Enrico H. Gerding \\ School of Electronics and Computer Science \\ University of Southampton, $U K$ \\ \{ama07r,dem,eg\}@ecs.soton.ac.uk
}

\section{Introduction}

In many educational organizations there exist restrictions on which courses might run, due to the overheads of running too many courses. Now, in the context of personalized learning, where students are taking more control of their learning, we would like the decision about which courses are run to be made collectively by the students, while taking into account their individual preferences. Multiagent systems are a powerful technology to tackle this complexity and to enable flexible course selection systems because of features such as autonomy, responsibility, social ability and intelligence $[1,2]$. In particular, voting systems can be used to reach a socially desirable decisions, while taking into account individual preferences [3]

In this paper, we use a voting protocol introduced in our previous work [4], which combines features from single transferable vote (STV) and cumulative voting [5]. Specifically, this protocol takes advantage of the features of cumulative voting to express the preferences using points and, at the same time, allows for multiple rounds to avoid wastage by allowing the transfer of points in a similar way to the transfer of votes in STV. In addition, we introduced a number of voting strategies that can be used by the student agents in the system. However, different from our previous work, where courses were independent, we extend this work by considering a more complex setting with combinatorial student preferences.

Combinatorial preferences have been mostly studied within the context of combinatorial auctions, although there are a couple of papers that consider such preferences in a voting setting $[6,7]$. In these works, agents are assumed to vote for given bundles of candidates. Since enumerating all possible combinations of candidates is typically infeasible, the main problem is then which of these bundles should be selected by the system and voted on by the agents. Our paper takes a different approach and uses a multi-round voting protocol, where in each round the candidate with the least number of votes is eliminated. Furthermore, it uses an agent-based approach where in both combinatorial voting and auctions there are a number of languages for representing preferences.
Some of these languages are tailored to represent cardinal preferences while others represent ordinal preferences. Expressing preferences that allows agents to specify preferences concisely and clearly include the OR-language and XOR-language from combinatorial auctions [8]. In this paper, we use the same principle as the languages discussed above, but limit the number of combinations only by consider interdependencies between two courses.

\section{The Multiagent System}

Figure 1 shows the entities and objects that form the system. Our system consists of a single university agent (UA) and several student agents (SAs). The SA contains the student's preferences (in our experiments we automatically generate these according to the parameters of the experiment) and also an appropriate voting strategy. Then the SAs and the UA use a voting procedure to interact with each other and determine which courses to run.

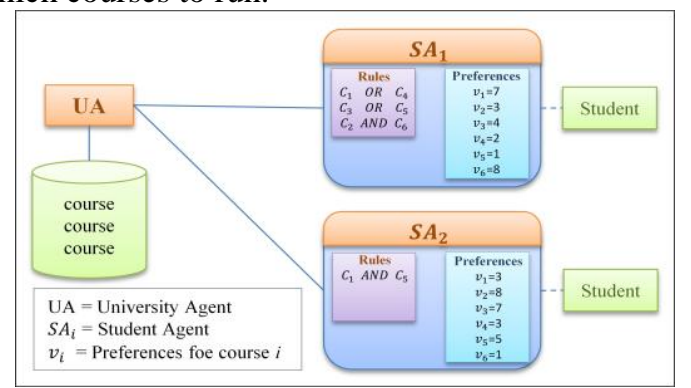

Figure 1. System architecture

The voting protocol proceeds in several rounds as follows. Each SA initially receives an equal and fixed number of points. In each round, each SA can allocate some or all of their available points to the available courses. After that, the UA cancels the course with the lowest cumulative points. Then, the UA refunds the points for the cancelled course and informs all the SAs about the cancelled course, and the current cumulative points allocated to the remaining courses (this information about how others have voted collectively can then be used by the agent's voting strategy to place votes in subsequent rounds). The protocol proceeds to the next round and SAs can vote again using their 
remaining points (this includes the refunded points and any points left in the previous rounds). The process is repeated until the desired number of courses is remaining. For more details see [4].

\subsection{Student Preferences}

The student preferences are modeled using a utility function. Importantly, the utility of a course may depend of which other courses are running. We have two types of relationship: complementary (AND) and substitute (OR). In more detail, two courses, A and B, are complementary when the student is only interested in choosing course $\mathrm{A}$ if course $\mathrm{B}$ is running, and vice versa. Conversely, courses $\mathrm{A}$ and $\mathrm{B}$ are substitutes if a student is interested in course A or course B, but not both. We assume that each student has a (possibly different) set of rules, where each represents either an AND or an OR relationship between a pair of courses.

Given this, the utility function of a student is modeled as follows. Let $C=\left\{C_{1}, C_{2}, \ldots, C_{m}\right\}$ denote the set of courses, and $\vec{v}=\left\{v_{1}, v_{2}, \ldots ., v_{m}\right\}$ the individual utilities (in case there are no rules) for these courses, where $m$ is the total number of available courses to choose from. Furthermore, let $R_{O R}$ denote the set of OR rules, which specifies a set of pairs of courses $\left(C_{i}, C_{j}\right)$ and, similarly, $R_{A N D}$ denotes the set of AND rules. To avoid conflicts, we assume that each course is only part of one rule. Therefore, the same course cannot appear both in an OR rule and an AND rule. Then, the utility for a set of running courses is calculated as follows. For any running course $C_{i}$ that does appear in a rule, the utility is simply the sum of the individual utilities $v_{i}$ of those courses. For any pair of courses $\left(C_{i}, C_{j}\right) \in R_{O R}$, if both courses are running, the utility for the pair is $\max \left(v_{i}, v_{j}\right)$. If only one of them is running, then the utility is equal to the individual utility for that course. For any pair of courses $\left(C_{i}, C_{j}\right) \in R_{A N D}$, if both courses are running, the utility for the pair is $v_{i}+v_{j}$. Otherwise, the utility is zero. The total utility is then the sum of the individual courses without rules, and the pairs with rules. Using this utility, we then calculate the student satisfaction by taking the utility as a percentage of the utility the student would achieve when all the courses would be running.

\subsection{Strategies}

In this paper we use the same strategies from [4] but extend the more advanced strategies to take the complex preferences into account.

Equal share: This strategy is used as a benchmark and simply allocates an equal number of votes to each course, regardless of the student's preference. Formally, the total number of points to be allocated to course $j$, is given by $b_{j}=R P / m$, where $R P$ denotes the total number of points remaining.

Proportional: This strategy allocates all the points proportionally to the utility for individual courses. Furthermore, the strategy takes the AND rule into account by not placing any votes if their counterpart has been cancelled. Formally, let $C^{\prime}$ denote the set of courses that are not cancelled, and that contain no courses from any AND rule where one of them is cancelled. Then, the total number of points to be allocated to course $j \in C^{\prime}$ is:

$$
b_{j}=\frac{R P}{\sum_{i \in C^{\prime}} v_{i}} \cdot v_{j}
$$

Intelligent: This more sophisticated strategy tries to predict the probability that a course will be cancelled based on the number of points currently allocated to each course from previous rounds. Furthermore, it considers the rules between courses when calculating this probability. This strategy does not spend all the points in the first round, in order to take advantage of the information that is received in subsequent rounds. Otherwise, it would only have the points returned from cancelled courses to use in these rounds. In the last voting round, it allocates all remaining points. Furthermore, in first round, because the strategy does not have any information about courses, it distributes half of the points using the proportional strategy as explained above.

The strategy tries to estimate the probabilities of courses being cancelled for a given allocation of points, using a softmax function [9]. Then, given these probabilities, it tries to estimate the expected utility for a given distribution of points. Finally, it uses a search algorithm to find the point allocation which maximizes expected satisfaction. Formally, the probability that a course $i$ is going to be cancelled is given by:

$$
P_{C A N C E L}\left(C_{i} \mid \vec{b}\right)=\frac{e^{\frac{-\left(\mathrm{cp}_{i}+\mathrm{b}_{i}\right)}{\sum_{\mathrm{j}=1}^{\mathrm{m}}\left(\mathrm{cp}_{j}+\mathrm{b}_{j}\right)} \cdot \beta}}{\sum_{k=1}^{m} e^{\frac{-\left(\mathrm{cp}_{k}+\mathrm{b}_{k}\right)}{\sum_{\mathrm{j}=1}\left(\mathrm{cp}_{j}+\mathrm{b}_{j}\right)} \cdot \beta}}
$$

Where $\mathrm{cp}_{i}$ is the cumulative number of points which have so far been allocated to course $C_{i}$ (including points from other agents), and $b_{i}$ is the number of points that the $\mathrm{SA}$ is planning to allocate to course $C_{i}$ in the current voting round, and $\vec{b}$ is the vector of points to be allocated. Furthermore, $\beta$ is a constant which enables a range of different strategies. For example, if $\beta=0$, then each course is equally likely to be cancelled, irrespective of the cumulative number of points currently allocated. At the other extreme, as $\beta \rightarrow \infty$, the course with the lowest total number of points will be cancelled with probability 1 , and all other courses will be cancelled with probability 0 .

We now show how we can use this probability to calculate the expected utility, EU, of an SA, given the 
vector of points, $\vec{b}$, and taking into account both AND and OR relationship. Suppose we have an AND relationship between $C_{1}$ and $C_{2}$. In order to calculate the expected utility, given the probability that both of these courses will run, i.e., $P_{R U N}\left(C_{1} \mid \vec{b}\right) \cap P_{R U N}\left(C_{2} \mid \vec{b}\right)$, where $P_{R U N}\left(C_{i} \mid \vec{b}\right)=1-P_{C A N C E L}\left(C_{i} \mid \vec{b}\right)$. Now, in the case of the OR relationship between $C_{1}$ and $C_{2}$, we need to consider also the possibility that only one of them will run, and the other is canceled. This gives the following expected utility for individual or pairs of courses, depending on the rules between courses:

- $\quad$ No rule: $E U=P_{R U N}\left(C_{i} \mid \vec{b}\right) * v_{i}$

- $C_{i} \operatorname{AND} C_{j}: E U=P_{R U N}\left(C_{i} \mid \vec{b}\right) * P_{R U N}\left(C_{j} \mid \vec{b}\right) *\left(v_{i}+v_{j}\right)$

- $C_{i} \mathrm{OR} C_{j}: E U=P_{R U N}\left(C_{i} \mid \vec{b}\right) * P_{R U N}\left(C_{j} \mid \vec{b}\right) *$ $\operatorname{MAX}\left(v_{i}, v_{j}\right)+P_{\text {RUN }}\left(C_{i} \mid \vec{b}\right) * P_{C A N C E L}\left(C_{j} \mid \vec{b}\right) * v_{i}+$ $P_{\text {CANCEL }}\left(C_{i} \mid \vec{b}\right) * P_{R U N}\left(C_{j \mid \vec{b}}\right) * v_{j}$

The total expected utility is then the sum of the utilities for individual courses without rules, and course pairs with rules.

The next step is then to find the allocation that maximises this expected utility. We used random sampling by randomly generating 1000 vectors $\vec{b}$ subject to the constraint that the total number of points is equal to the number of points that we would like to spend in the current round. We then take the allocation with the highest expected satisfaction.

\section{Evaluation}

In this section, we evaluate the voting procedure and explore the impact of the three strategies described above on the overall student satisfaction. We consider 3 different scenarios. Table 1 shows the settings for these cases of different scenarios (for more details see[4] ). We run each scenario 30 times with different randomly generated student preferences, to obtain statistically significant results.

Table 1. Different setting to the scenarios

\begin{tabular}{|c|c|c|c|}
\hline Scenario & \#courses $(\mathrm{m})$ & \#running courses (r) & \#students (n) \\
\hline 1 & 51 & 40 & 100 \\
\hline 2 & 33 & 11 & 60 \\
\hline 3 & 18 & 9 & 20 \\
\hline
\end{tabular}

\subsection{Student Agent Preferences}

For each simulation run, we generate the student preferences as follows. We first randomly generate preferences for individual courses from a uniform distribution between 0 and 10 . Then, we generate the AND and OR relationships as follows. First, we generate all possible AND and OR relationships. Then, we select a subset of these rules from which the students can select. We do this to increase the likelihood that groups of students have similar relationships between courses. This is true in practice, since often students have the same relationships between courses, even if they value the individual courses differently. We can vary the degree of similarity by changing the size of the subset as a percentage of the total number of rules.

\subsection{Analysis}

We compare the case where a proportion of the students use one strategy, and the remainder of the students uses another strategy. In the results that follow, the y-axis shows the student satisfaction for each group of agents using a particular strategy, as well as the overall average satisfaction. Furthermore, on the $\mathrm{x}$-axis we vary the proportion of students using a particular strategy for different percentage of applied rules. For example, in figures 2 and 3, 10-90 means that 10 students use the proportional strategy, and 90 students use the equal share strategy and this is repeated for three different settings: NO rules, 50\% rules and $100 \%$ rules. The errorbars show the $95 \%$ confidence intervals.

The results in figures 2 and 3 show that the intelligent and proportional strategies are both clearly better than the equal share. Moreover, as the number of rules increases, the better the intelligent and proportional strategies perform. On average, the improvement is around $4 \%, 6 \%, 9 \%$, for NO rules, $50 \%$ and $100 \%$ rules respectively. Furthermore, the average satisfaction of all students also increases, which means that the allocation is more efficient when students use a more intelligent voting approach. The results for other scenarios are very similar and not shown to avoid repetition.

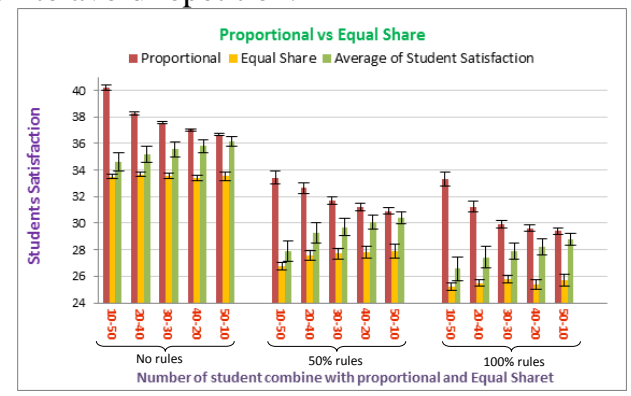

Figure2. Scenario 2: Proportional vs. Equal Share

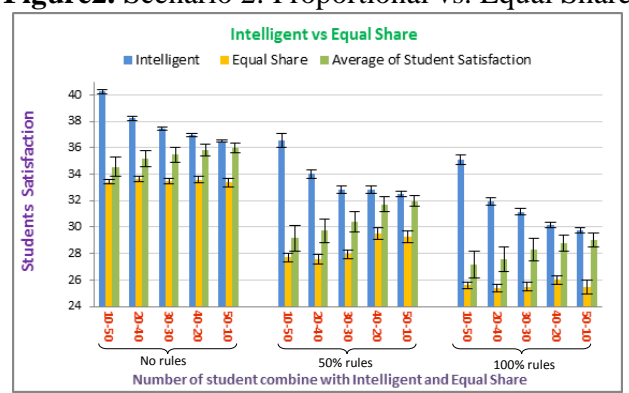

Figure 3. Scenario 2: Intelligent vs. Equal Share 
Figures 4, 5 and 6 compare the results using the intelligent strategy and the proportional strategy for the 3 different scenarios. Figure 4 shows that, at first glance, the performance of intelligent strategy increases, comparing to proportional strategy, as the students apply more rules. However, in most cases this result is not statistically significant. The reason is that, first, in scenario 1, the number of courses that the students vote over is large. This means that the range of student choice is wide, and students have a wide range of preferences. Second, the number of students voting is also large. This means that each individual student has very little voting power. To analyze this, we now consider a setting where the number of students and courses are small.

Figures 5 and 6 show that, with relatively few courses and students, there are clear differences in the performance of the intelligent and proportional strategies. The intelligent strategy significantly outperforms proportional strategy when student apply rules and this superiority is increased as more rules are applied. This holds in almost all cases where rules were applied, according to a t-test with $95 \%$. This suggests that, when students have fewer choices and therefore there is less differentiation between the students, and when the number of students is not too large, the intelligent strategy perform better than proportional. Note also that, as the proportion of students using the intelligent strategy increases, the student satisfaction of all students either stays the same, or increases. Therefore, using a more intelligent approach does not harm the system as a whole.

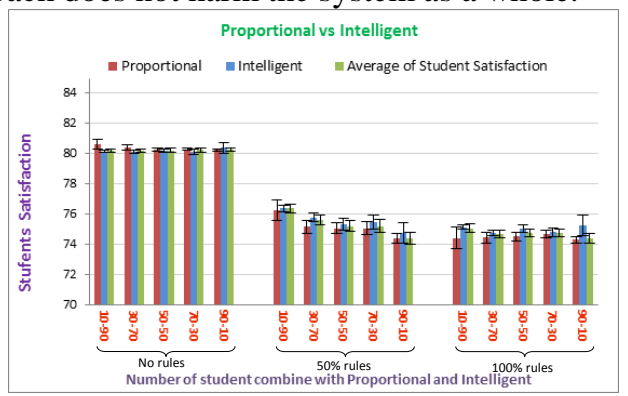

Figure 4. Scenario 1: Proportional vs. Intelligent

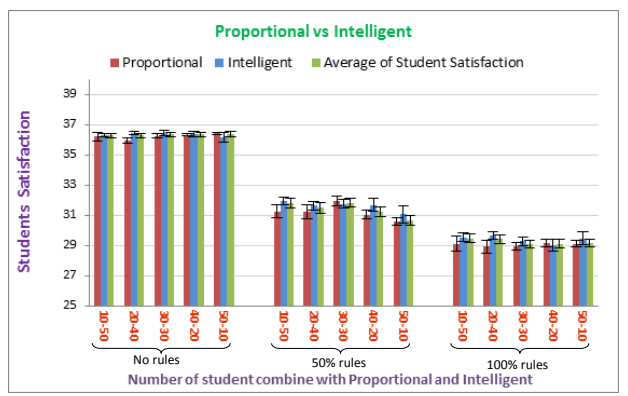

Figure 5. Scenario 2: Proportional vs. Intelligent

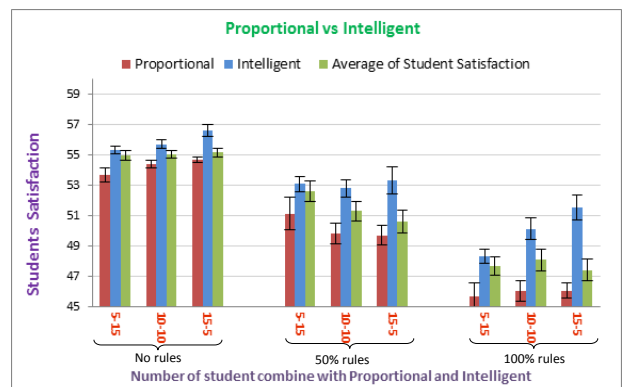

Figure 6. Scenario 3: Proportional vs. Intelligent

\section{Conclusion}

We presented a multiagent system for course selection based on voting theory, where students have complementary and substitutable preferences between courses. We also developed a number of voting strategies that student agents could use to place votes on a student's behalf. We found that, when students have complex preferences and the number of students is not too large such that each individual student can affect the voting outcome, the intelligent strategy performs significantly better than proportional.

Our future work consists of two parts. First of all, we intend to apply fairness principals to the voting outcome. Furthermore, we intend to consider other intelligent voting strategies and alternative voting procedures, and explore constraints such as limitations on the number of courses that a student can take, and having pre-requisite courses.

\section{References}

[1] Wang, J., Sun, Y.-H., Fan, Z.-P. and Liu, Y. A Collaborative Elearning System Based on Multi-agent. Springer Berlin / Heidelberg, City, 2005.

[2] Jennings, N. R. and Wooldridge, M. Applications of intelligent agents. Springer-Verlag New York, Inc., City, 1998.

[3] Wooldridge, M. J. An introduction to multiagent systems. Wiley ; Chichester, Hoboken, N.J., 2009.

[4] Ali, M. A., Enrico, H. G. and David, E. M. A Voting-Based Agent System for Course Selection in E-Learning. IEEE/WIC/ACM International Conference on Intelligent Agent Technology2010), 303-310.

[5] Shoham, Y. and Leyton-Brown, K. Multiagent Systems: Algorithmic, Game-Theoretic, and Logical Foundations. Cambridge University Press, 2008.

[6] Lang, J. Logical Preference Representation and Combinatorial Vote. Annals of Mathematics and Artificial Intelligence, 42, 1-3 2004), 37-71.

[7] Lang, J. Voting in Combinatorial Domains: What Logic and AI Have to Say. Springer Berlin - Heidelberg, City, 2008.

[8] Uckelman, J. and Endriss, U. Winner determination in combinatorial auctions with logic-based bidding languages. Proceedings of the 7th international joint conference on Autonomous agents and multiagent systems - Volume 32008), 1617-1620.

[9] Hensher, D. A., Rose, J. M. and Greene, W. H. Applied choice analysis : a primer. Cambridge University Press, Cambridge, 2005. 